\title{
THE REVERSE HARDY INEQUALITY WITH MEASURES
}

\section{W. Desmond Evans, Amiran Gogatishvili And Bohumír Opic}

Abstract. In this paper we characterize the validity of the inequalities

$$
\|g\|_{p,(a, b), \lambda} \leqslant c\left\|u(x) \int_{(a, x)} g(y) d \mu\right\|_{q,(a, b), v}
$$

and

$$
\|g\|_{p,(a, b), \lambda} \leqslant c\left\|u(x) \int_{(x, b)} g(y) d \mu\right\|_{q,(a, b), v}
$$

for non-negative Borel measurable functions $\mathrm{g}$ on the interval $(a, b) \subseteq \mathbb{R}$, where $0<p \leqslant 1$, $0<q \leqslant+\infty, \lambda, \mu$ and $v$ are non-negative Borel measures on $(a, b)$, and $u$ is a weight function on $(a, b)$.

Mathematics subject classification (2000): 26D10, 26D15, 46E30.

Key words and phrases: Hardy operator, reverse Hardy inequality, Borel measures, weight functions.

\section{REFERENCES}

[1] P. R. BeEsack, H. P. HeInIG, Hardy's inequalities with indices less than 1, Proc. Amer. Math. Soc., 83 (1981), 532-536.

[2] G. BennetT, Factorizing the classical inequalities, Mem. Amer. Math. Soc., 120 (1996), no. 576.

[3] G. A. BLISS, An integral inequality, J. London Math. Soc., 5 (1930), 40-46.

[4] E. T. Copson, Note on a series of positive terms, J. London Math. Soc., 2 (1927), 9-12.

[5] E. T. Copson, Note on a series of positive terms, J. London Math. Soc., 3 (1928), 49-51.

[6] A. GogatishVILI, L. PICK, Discretization and anti-discretization of rearrangement-invariant norms, Publ. Mat., 47, (2003) 311-358.

[7] K.-G. Grosse-Erdmann, The Blocking Technique, Weighted Mean Operators and Hardy's Inequality, Lect. Notes Math. 1679, Springer, Berlin, 1998.

[8] G.H. HARDY, Note on a theorem of Hilbert, Math. Zeitschr., 6 (1920), 314-317.

[9] A. Kufner, L.-E. Persson, Weighted inequalities of Hardy type, World Scientific Publishing Co, Singapore, 2003.

[10] E. LANDAU, A note on a theorem concerning series of positive terms, J. London Math. Soc., 1 (1926), 38-39.

[11] L. LeINDLER, Inequalities of Hardy and Littlewood type, Acta Sci. Math. (Szeged), 2 (1976), 117-123.

[12] L. LEINDLER, On the converses of inequalities of Hardy and Littlewood, Acta Sci. Math. (Szeged), 58 (1993), 191-196.

[13] B. OpIC, A. Kufner, Hardy-type inequalities, Pitman Reaserch Notes in Mathematics Series, 219, Longman Scientific \& Technical, Harlow, 1990.

[14] D. V. PROKHOROV, Weighted Hardy's inequalities for negative indices, Publ. Mat., 48 (2004), $423-443$.

[15] W. Rudin, Principles of Mathematical Analysis, McGraw-Hill Book Company, New York, 2nd ed., 1964.

[16] E. SAWYER, Weighted Lebesgue and Lorentz norm inequalities for the Hardy operator, Trans. Amer. Math. Soc., 281 (1984), 329-337. 